\title{
AN ALIASING DETECTION ALGORITHM BASED ON SUSPICIOUS COLOCALIZATIONS OF FOURIER COEFFICIENTS
}

\author{
Baptiste Coulange and Lionel Moisan \\ Université Paris Descartes, MAP5, CNRS UMR 8145, Paris, France
}

\begin{abstract}
We propose a new algorithm able to detect the presence and the localization of aliasing in a single digital image. Considering the image in Fourier domain, the fact that two frequencies in aliasing relation contribute to similar parts of the image domain is a suspicious coincidence, that we detect with an a-contrario model. This leads to a localization of the aliasing phenomenon in both spatial and spectral domains, with a detection algorithm that keeps control of the number of false alarms. Experiments on several images show that this new method favorably compares to the state of the art, and opens interesting perspectives in terms of image enhancement.
\end{abstract}

Index Terms - Sampling, aliasing, Fourier transform, acontrario detection.

\section{INTRODUCTION}

In theory, signal or image sampling is a simple operation, well understood thanks to Shannon's Sampling Theorem. In practice however, the required ideal low-pass filter (sinc convolution) is physically unreachable, and the required presampling filter has to reach a compromise between aliasing (due to the fact that frequencies above the Nyquist limit are only attenuated) and blur (caused by the attenuation of frequencies under the Nyquist limit), as proposed in [8] for example. Thus, aliasing is a necessary evil, and understanding better the possibilities to detect and correct it could play an essential role in the global optimization of image acquisition processes and lead to significant improvements of the quality of reconstructed images while keeping the sampling rate unchanged.

The aliasing phenomenon has three main visual effects: an effect on textures (a high-frequency texture may be transformed into a totally different low-frequency one), an effect on thin structures (a continuous line may be transformed into a dotted one), and an effect on edges (that generally present a staircase aspect after aliasing). From a more quantitative point of view, the main loss caused by aliasing is the ability to reach a good sub-pixel precision using image interpolation (ideally, sinc interpolation).

Aliasing detection and/or correction is decisive in applications where sub-pixel precision is concerned, in particular satellite imaging (photo-interpretation), disparity estimation (and other correlation-based techniques), local orientation estimation, etc. Moreover, a general-purpose method able to detect well-sampled images (well-sampled meaning here "sampled without aliasing") in a no-reference framework is useful for generic image-processing tools (e.g. integrated softwares or on-line image processing demos) that have to deal with images whose origin may not be precisely known. Last, the present work may be useful for image quality assessment, though we do not restrict ourselves to visible aliasing here.

The issue of aliasing detection in a single image has not been considered much in the literature. The problem we consider here is similar to the one addressed by Reibman and Suthaharan [7], except that we focus on aliasing detection itself rather than on the construction of an image quality metric. Other contributions exist on aliasing detection in different frameworks, in particular in the case of multiple images.

In this paper, we propose to detect aliasing by using the fact that many image structures contribute to the image spectrum in specific locations (leading, typically, to a main localized contribution around a given fundamental frequency and several secondary contributions around harmonics, that is, frequencies that are multiple of the fundamental frequency). If an harmonic is aliased but the fundamental frequency is not, then the fact that these two frequencies contribute to the same image parts may be detected as a suspicious coincidence. In Section 2, we describe how to measure the relative impact of a given frequency zone on the image domain using a non-symmetric bandpass filter. This enables us to build an aliasing detection algorithm (Section 3), based on an a-contrario formulation [2] and a post-processing step that guarantees that no detection will be found in random data. The generic algorithm we obtain, that has a single parameter (the expected number of false alarms), is evaluated on several images in Section 4 and compared to [7], before we conclude by showing interesting perspectives in terms of aliasing removal and resolution improvement.

\section{LOCALIZATION OF FOURIER COEFFICIENTS}

Let $u: \Omega \rightarrow \mathbb{R}$ be a discrete gray-level image, where $\Omega=$ $\{0, \ldots, n-1\}^{2}$ is the image domain (we assume that $\Omega$ is a square to simplify notations) and $u(\mathbf{x})$ represents the inten- 
sity of a pixel $\mathbf{x} \in \Omega$. The Discrete Fourier Transform of $u$ is the complex image $\hat{u}: R \rightarrow \mathbb{C}$ defined by $\hat{u}(\mu)=$ $\sum_{\mathbf{x} \in \Omega} u(\mathbf{x}) e^{\frac{-2 i \pi}{n}<\mathbf{x} \mid \mu>}$ where $R=\mathbb{Z}^{2} \cap\left[-\frac{n}{2}, \frac{n}{2}\right)^{2}$ is the spectral domain. If we want to measure the contribution of frequencies around $\xi \in R$ to the reconstruction of $u$, we can choose a symmetric localization function $g: \mathbb{Z}^{2} \rightarrow \mathbb{R}$ and consider the band-pass filtered image

$\mathcal{F}_{\xi}^{g}(u)(\mathbf{x})=\frac{1}{n^{2}} \sum_{\mu \in R}(g(\mu+\xi)+g(\mu-\xi)) \hat{u}(\mu) e^{\frac{2 i \pi}{n}<\mathbf{x} \mid \mu>}$

where we assume that the support of $g$ is small enough to ensure that $\xi$ belongs to the set

$$
S_{g}=\{\xi \in R, \forall \mu \in R, g(\mu-\xi) \cdot g(\mu+\xi)=0\} .
$$

In the following, the functions $g$ we shall use will be indicator functions of squares, centered in 0 , but smoother functions could be used as well. The problem with the image $\mathcal{F}_{\xi}^{g}(u)$ is that by construction, it locally oscillates and cannot be used directly to measure the importance of the contribution in each pixel. To get rid of this phenomenon, we propose to use the notion of analytic part, originally introduced for one-dimensional signals by Gabor [3], and then generalized in several ways to 2D images (e.g. [4]). In the case we consider, this leads to the following

Definition 1. The analytic part of an image $u$ associated to a window $(\xi, g)$ is

$$
u_{\xi}^{g}(\mathbf{x})=\frac{1}{n^{2}} \sum_{\mu \in R} g(\mu+\xi) \hat{u}(\mu) e^{\frac{2 i \pi}{n}<\boldsymbol{x} \mid \mu>} .
$$

The interest of considering the analytic part $u_{\xi}^{g}$ is that its real part is exactly the band-pass filtering $\left(\mathcal{F}_{\xi}^{g}(u)=\operatorname{Re}\left(u_{\xi}^{g}\right)\right)$, but its modulus $\left|u_{\xi}^{g}\right|$ measures the amplitude of the oscillations, that bounds $\left|\mathcal{F}_{\xi}^{g}(u)\right|$ from above everywhere. The difference is illustrated on Fig. 1. Notice by the way that the square modulus of the analytic part is expected to be very smooth, because $g$ has a small support. Indeed, if $\operatorname{supp}(g) \subset$ $[-k, k]^{2}$, then

$$
\forall \xi \in S_{g}, \quad \operatorname{supp} \widehat{\left|u_{\xi}^{g}\right|^{2}} \subset[-2 k, 2 k]^{2} .
$$

\section{DETECTING SUSPICIOUS COLOCALIZATIONS}

In this section, we build an aliasing detection algorithm that consists in two steps. To simplify the analysis, we consider the simplest case of aliasing, with a sub-sampling factor of 2 , but hypotheses on the initial spectral domain corresponding to Quincunx sampling [5] as in SPOT 5 satellite. In this configuration, the aliasing phenomenon has the property that each Fourier coefficient of the sampled image can be written

$$
\hat{u}(\xi)=\hat{v}(\xi)+\hat{v}(H(\xi / 2))
$$

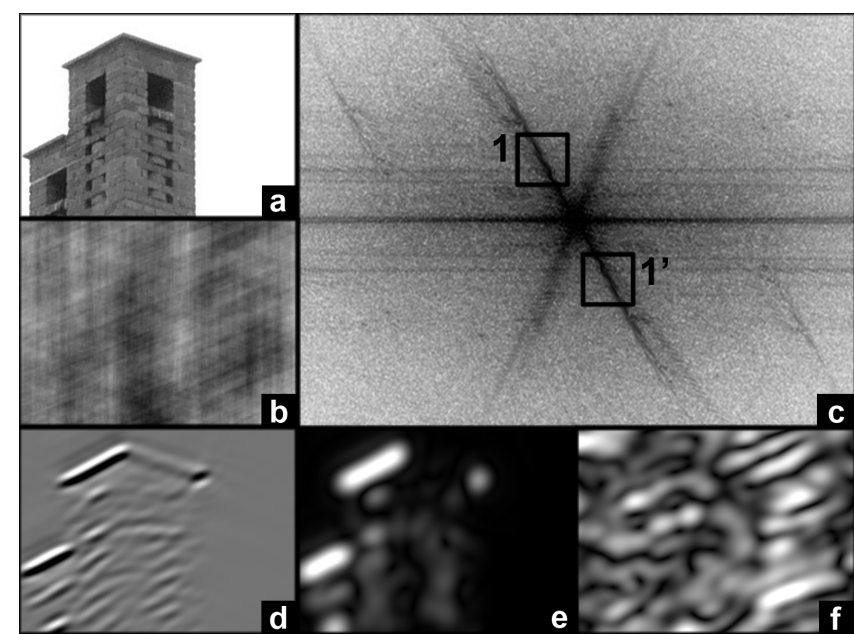

Fig. 1. Analytic part. Images $a$ and $b$ have the same Fourier modulus (represented in $c$ ), but image $b$ has random phases. Image $d$ corresponds to image $a$ after a pass-band filter whose spectral support is localized in squares 1 and $1^{\prime}$. Image $e$ (resp. $f$ ) is the modulus of the analytic part of $a$ (resp. b), corresponding to square 1 alone. All Fourier visualizations in this article are treated by the periodic plus smooth decomposition to avoid periodization artifacts [6].

where $v$ is the original image (before sampling) and $H$ is the one-to-one function that associates to an observed frequency $\xi \in R$ the unique alias of $2 \xi$ in $R$ modulo $R$ (see Fig. 2).

The first step of aliasing detection consists in finding frequencies $\xi$ for which the energy of the maps $\left|u_{\xi}^{g}\right|^{2}$ and $\left|u_{H(\xi)}^{g}\right|^{2}$ are localized spatially in similar domains. Since the values of these two maps may be very different (the energy in Fourier domain quickly decreases as the frequency increases), we propose to detect the similarities between these two maps in a relative way, by comparing their cross-correlation with the cross-correlations obtained after applying a shift to one of these maps.

Definition 2. Given a $2 D$ shift vector $\eta \in \mathbb{Z}^{2}$, We define the analytic correlation map associated to a frequency window $(g, \xi)$ as the function

$$
\eta \mapsto C_{g, \xi}(\eta)=\sum_{x \in \Omega}\left|u_{\xi}^{g}(\mathbf{x}) \cdot u_{H(\xi)}^{g}(\mathbf{x}-\eta)\right|^{2},
$$

with the convention that all analytic parts are $\Omega$-periodic.

Now considering the smallest shift(s) $\eta_{0}$ (for the $|\cdot|_{\infty}$ norm) for which the $\Omega$-periodic function $\eta \mapsto C_{g, \xi}(\eta)$ attains its maximum value, we define $r(\xi)=\left|\eta_{0}\right|_{\infty}$, and propose to use an a-contrario model [2] to detect small values of $r(\xi)$ against the null hypothesis $\left(H_{0}\right)$ that all possible $n^{2}$ locations of $\eta$ are equally likely. In this framework, we detect aliasing as a contradiction of $H_{0}$, with the statistical test $N F A(w) \leq$ $\varepsilon$, where

$$
N F A(w)=|W| \cdot \frac{(2 r(\xi)+1)^{2}}{n^{2}},
$$




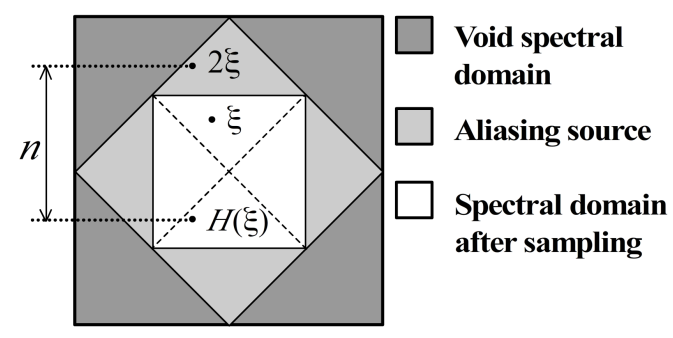

Fig. 2. The quincunx hypothesis consists in assuming that the original image spectrum is null in the darkest region above. After a sub-sampling by a factor 2 , each frequency of the final spectral domain (in white) is corrupted by a single aliased frequency coming from the "aliasing source" domain.

$w=(\xi, g)$ is a frequency window (associated to a central frequency $\xi$ and a localization function $g$ ), and $W$ the set of all such windows we test (we shall comment on the choice of $W$ later). This definition ensures that $N F A$ is a number of false alarms, in the following sense:

Proposition 1. If $u$ is a random image such that the probability of any considered analytic correlation map is invariant under periodic translation, then the expectation of the number of windows $w \in W$ such that $N F A(w) \leq \varepsilon$ is less than $\varepsilon$.

Note that the hypothesis we make in Proposition 1 applies in particular to the case when $U$ is a random phase image, that is, an image whose Fourier transform has a known modulus and random uniform independent phases (up to the symmetry constraint).

The a-contrario model above detects suspicious colocalization between Fourier zones in aliasing relation (in the sense that they contradict the $H_{0}$ hypothesis), but the detection of some windows may also be caused by textured zones with a rich and isotropic spectral content. In order to get rid of these undesired detections, we hence propose a post-processing step. It consists in selecting, among the windows detected in the first step, only those for which the maximum value of the analytic correlation map in the domain $[-r(\xi), r(\xi)]^{2}$ does not increase when considering other central frequencies $\xi^{\prime}$ that could be in aliasing relation with $H(\xi)$ for a different sampling rate. It is not difficult to see that the corresponding frequencies all belong to a vertical (or horizontal) line passing through $\xi$.

In the end, the two-steps aliasing detection algorithm we just presented yields, if the image is detected as aliased, a certain number of square windows in Fourier domain. Let us call aliasing zone any connected component of the union of these windows. For each aliasing zone, we define a normalized correlation image (with maximum value equal to 1 ), that can be used as a mask in spatial domain, both to evaluate the aliased part of the image domain and to try to reverse the aliasing process, as shown in Section 4.

The algorithm we implemented uses windows defined by indicator functions of squares whose sidelength are of the kind $13+6 k, k=0 . .5$. For each window size, we chose window locations in order to ensure an overlap of half a window for adjacent windows. The detection threshold on the NFA function was, as usually done, set to 1 .

\section{PERFORMANCE RESULTS}

We tested the proposed algorithm on several images, in two different situations. On one hand, we produced "wellsampled" images by reducing the original $512 \times 512$ images to $256 \times 256$ with a perfect low-pass filter (hard frequency cutoff). On the other hand, we cleared the highest frequencies (dark zone in Fig. 2) of the original $512 \times 512$ images, and sampled them by a factor two to produce "quincunx-aliased" images. These two series of images were then analyzed separately, both with the SAM method [7] and ours. The results are reported in Table 1.

\begin{tabular}{|l|c|c|c|c|c|c|}
\hline image & \multicolumn{2}{|c|}{ well-sampled } & \multicolumn{4}{|c|}{ aliased } \\
& nd & SAM & nd & $\%$ S & $\%$ F & SAM \\
\hline pipe & 0 & 25.3 & 3 & 16.2 & 2.9 & 23.0 \\
\hline barbara & 1 & 19.2 & 10 & 1.6 & 5.4 & 18.4 \\
\hline lena & 0 & 16.6 & 2 & 7.2 & 1.3 & 16.4 \\
\hline harbor [7] & 0 & 18.1 & 8 & 1.5 & 5.5 & 16.1 \\
\hline bridge [7] & 0 & 17.1 & 5 & 11.2 & 1.6 & 15.2 \\
\hline boat & 0 & 15.5 & 7 & 4.3 & 10.4 & 15.1 \\
\hline butokai & 0 & 20.5 & 34 & 7.1 & 12.8 & 14.7 \\
\hline
\end{tabular}

Table 1. Results of aliasing detection. The algorithm we propose is compared to [7] on several images, in the case of well-sampled images and in the case of aliased images generated by sub-sampling. For the method we propose, "nd" corresponds to the number of detected aliased windows, " $\% S$ " is the relative area of the aliased spatial domain, and " $\% F$ " is the relative area of the aliased spectral domain.

These results call for several comments. First, as expected, no aliasing was detected for well-sampled images except for the barbara image that present a very special texture made of two fundamental frequencies in aliasing relation. Moreover, all aliased images we considered were systematically detected as such. However, considering the small number of detections for some of them (in particular pipe and lena), one could probably select parts of these images for which no aliasing would be detected. The method we propose offers several advantages compared to [7]. First, it tries to answer to the question "Does this image contains aliasing?", while Reibman and Suthaharan give an aliasing metric that is difficult to interpret on an absolute scale (though, as can be noticed in Table 1, their metric systematically decreases when aliasing is introduced). Another advantage of the method we propose is that it also permits to localize aliasing both in the frequency domain (Fig. 3) and in the spatial domain (Fig. 4). 
As another validation test, we also ran the present algorithm on the caps image from the LIVE database [9]. We detected aliasing on several places, and in particular on the right border of the up-left cap, exactly in the zone found by a completely different method [1].

In Fig. 5, we used the normalized correlation image defined at the end of Section 3 to enhance two aliased images, by reversing (with a spectral translation) the aliasing process on the analytic parts that were detected as aliased. This image enhancement algorithm is only a preliminary work that is not able to remove aliasing completely (even in the zone where aliasing is detected), but it open interesting perspectives for possible continuations of this work.

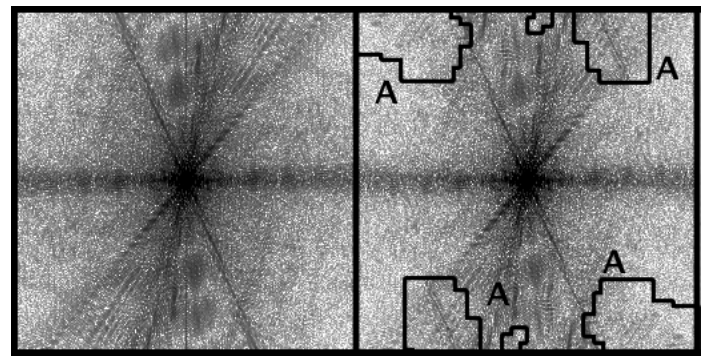

Fig. 3. Spectral aliasing detection. Fourier spectrum of the well-sampled version (left) and the aliased version (right) of the butokai image, superimposed with the detected aliased domains ("A" zone).

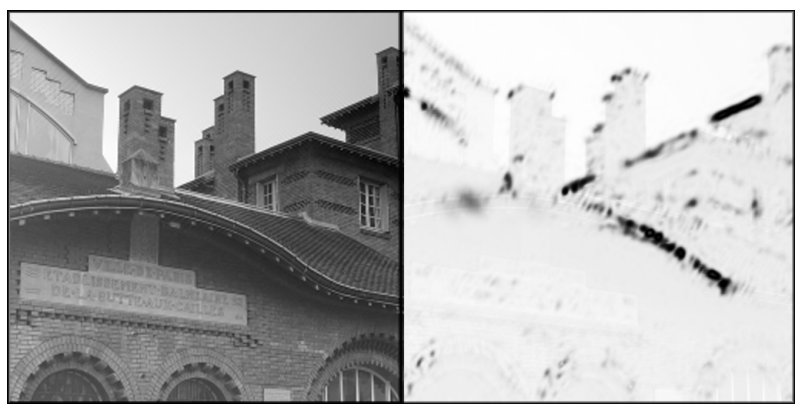

Fig. 4. The butokai image (left) and the spatial localization of the detected aliasing (in black on the right).

\section{CONCLUSION}

We presented a new generic algorithm able to detect aliasing in a digital image without access to a reference image. The proposed method achieves good detection results on artificially aliased images, while generally avoiding false detections on well-sampled ones. It also permits to localize the aliasing phenomenon, both in the spatial and in the spectral domains. The new theoretical approach we introduced to detect suspicious co-localization of Fourier coefficients brings interesting perspectives in terms of aliasing removal and resolution enhancement.

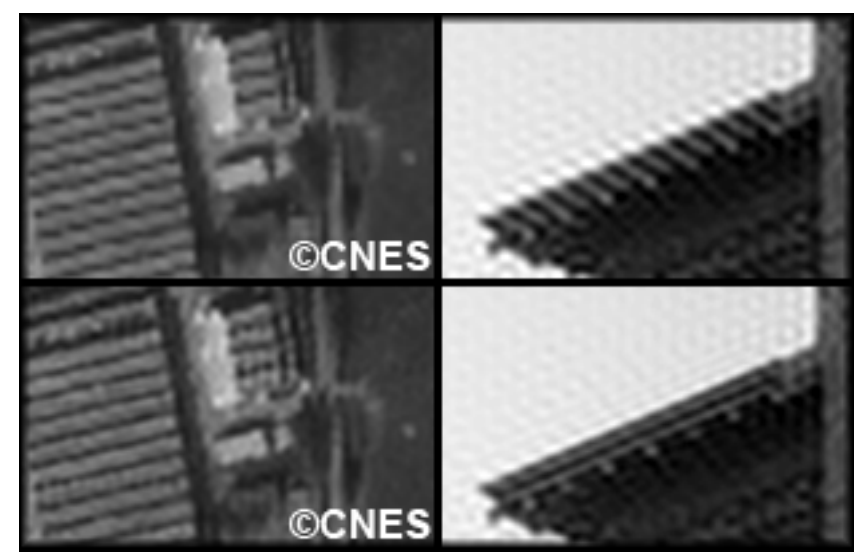

Fig. 5. Aliasing correction. First row: parts of two aliased images, the Nimes CNES image (left) and the butokai image (right). Second row: image obtained after detecting aliasing and translating back aliased space-frequency components in their estimated original places.

Acknowledgments. We thank Amy Reibman for providing us the harbor and bridge images used in [7], and the Centre National d'Études Spatiales (CNES) for supporting this work.

\section{REFERENCES}

[1] G. Blanchet, L. Moisan, B. Rougé, "Automatic detection of well sampled images via a new ringing measure", Int. Conf. on Acoustics, Speech and Signal Processing, 2010.

[2] A. Desolneux, L. Moisan, J.-M. Morel, From Gestalt Theory to Image Analysis: A Probabilistic Approach, Springer, 2008.

[3] D. Gabor, "Theory of communication", Journal of the Institute of Electrical Engineers, vol. 93, pp. 429-457, 1946.

[4] J.W. Havlicek, A.C. Bovik, "The Analytic Image", Int. Conf. on Image Processing, vol. 2, pp. 446-449, 1997.

[5] C. Latry, B. Rougé, "Super resolution: quincunx sampling and fusion processing", Int. Geoscience and Remote Sensing Symposium, pp. 315-317, 2003.

[6] L. Moisan, "Periodic plus smooth image decomposition", preprint MAP5, 2009.

[7] A.R. Reibman, S. Suthaharan, "A no-reference spatial aliasing measure for digital image resizing", Int. Conf. on Image Processing, pp. 1184-1187, 2008.

[8] A. Said, "A new class of filters for image interpolation and resizing", Int. Conf. on Image Processing, vol. 4, pp. 217-220, 2007.

[9] H.R. Sheikh, Z.Wang, L. Cormack, A.C. Bovik, "LIVE image quality assessment database release 2", http://live.ece.utexas.edu/research/quality. 